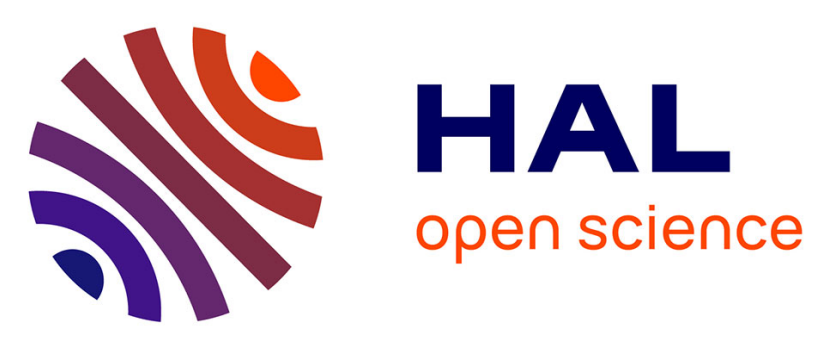

\title{
Microplastiques en Seine dans l'agglomération parisienne: étude des variations spatiales et temporelles des fibres anthropiques synthétiques et artificielles
}

Rachid Dris, Johnny Gasperi, Vincent Rocher, Bruno Tassin

\section{- To cite this version:}

Rachid Dris, Johnny Gasperi, Vincent Rocher, Bruno Tassin. Microplastiques en Seine dans l'agglomération parisienne: étude des variations spatiales et temporelles des fibres anthropiques synthétiques et artificielles. Techniques Sciences Méthodes , 2018, 5, pp.45 - 54. 10.1051/tsm/201805045 . hal-01803370

\section{HAL Id: hal-01803370 \\ https://hal-enpc.archives-ouvertes.fr/hal-01803370}

Submitted on 20 Jun 2018

HAL is a multi-disciplinary open access archive for the deposit and dissemination of scientific research documents, whether they are published or not. The documents may come from teaching and research institutions in France or abroad, or from public or private research centers.
L'archive ouverte pluridisciplinaire HAL, est destinée au dépôt et à la diffusion de documents scientifiques de niveau recherche, publiés ou non, émanant des établissements d'enseignement et de recherche français ou étrangers, des laboratoires publics ou privés. 


\title{
Microplastiques en Seine dans l'agglomération parisienne : étude des variations spatiales et temporelles des fibres anthropiques synthétiques et artificielles
}

\author{
Rachid DRIS $^{1 *}$, Johnny GASPERI ${ }^{1 *}$, Vincent ROCHER ${ }^{2}$ \\ , Bruno TASSIN ${ }^{1}$ \\ ${ }^{1}$ Université Paris-Est - Laboratoire eau environnement et systèmes urbains (LEESU) - 61, \\ avenue du Général de Gaulle - 94010 Créteil cedex. \\ ${ }^{2}$ Syndicat interdépartemental pour l'assainissement de l'agglomération parisienne \\ (SIAAP) - Direction du développement et de la prospective - 82 avenue Kléber - 92700 \\ Colombes. \\ 마isr@leesu.enpc.fr - gasperi@u-pec.fr
}

\section{Résumé}

Les fibres anthropogéniques sont abondamment présentes dans notre quotidien. Elles peuvent être naturelles, artificielles (cellulose régénérée) ou synthétiques (formées de matériaux pétrochimiques). Leur omniprésence conduit inévitablement à une contamination élevée dans l'environnement. Les études précédentes se concentrent sur les particules de plastique de taille inférieure à $5 \mathrm{~mm}$ (microplastiques) quelle que soit leur forme. Au contraire, cette étude examine les fibres exclusivement, sachant que ces dernières sont les plus ubiquitaires et représentent un risque d'ingestion plus élevé par les organismes. Un filet à maillage de $80 \mu \mathrm{m}$ est utilisé pour l'échantillonnage. Dans un premier temps, la variabilité temporelle à court terme des fibres en rivière a été déterminée. Lors d'un prélèvement d'une minute, un coefficient de variation de $45 \%(\mathrm{n}=6)$ a été obtenu alors qu'il passe à seulement $26 \%(\mathrm{n}=6)$ lors d'un prélèvement de 3 minutes. La répartition des fibres le long de la section de la rivière a aussi été identifiée et montre une possible différence des concentrations entre les berges et le centre. Ceci-dit, une absence de stratification verticale des concentrations a été observée. Un suivi mensuel des fibres a permis d'estimer une concentration moyenne de 100,6 $\pm 99,9$ fibres. $\mathrm{m}^{-3}$ dans la Marne, ainsi que des concentrations de 48,5 $\pm 98,5,27,9 \pm 26,3,27,9 \pm 40,3$ et 22,1 $\pm 25,3$ fibres. $\mathrm{m}^{-3}$, sur quatre points de la Seine, en allant de l'amont vers l'aval. Des flux de fibres ont été estimés à partir de ces concentrations mais aucun impact significatif de l'agglomération Parisienne n'a pu être mis en évidence. La présence de puits encore non étudiés, tel que la sédimentation, est suspectée. Cette étude démontre l'importance de considérer les fibres séparément des autres types de microplastiques, ainsi que la pertinence d'une approche considérant toutes les fibres, synthétiques ou non.

Mots clés : fibres, microplastiques, Seine, agglomération parisienne, plastiques 


\begin{abstract}
Processed fibers are highly present in our daily life and can be either natural, artificial (regenerated cellulose) or synthetic (made with petrochemicals). Their widespread use leads to a high contamination of the environment. Previous studies focus on plastic particles regardless of their type or shape as long as they are smaller than $5 \mathrm{~mm}$. On the contrary, this study focuses exclusively on fibers using a smaller mesh size net $(80 \mu \mathrm{m})$ to sample freshwater. First, the short term temporal variability of the fibers in the environment was assessed. While exposing the sampling net during 1 minute, a coefficient of variation of approx. $45 \%$ (with $\mathrm{n}=6)$ was determined. It was of only $26 \%(\mathrm{n}=6)$ when the exposure was of 3 minutes. The assessment of the distribution through the section showed a possible difference in concentrations between the middle of the water surface and the river banks which could be attributed to the intense river traffic within the Paris Megacity. The vertical variability seems negligible as turbulence and current conditions homogenize the distribution of the fibers. A monthly monitoring showed concentrations of $100.6 \pm 99.9$ fibers. $\mathrm{m}^{-3}$ in the Marne River and of $48.5 \pm 98.5,27.9 \pm 26.3,27.9 \pm 40.3$ and $22.1 \pm 25.3$ fibers. ${ }^{-3}$ from the upstream to downstream points in the Seine River. Once these concentrations are converted into fluxes, it seems that the impact generated by the Paris Megacity cannot be distinguished. Investigations on the role of sedimentation and deposition on the banks are required. This study helped fill some major knowledge gaps regarding the fibers in rivers, their sampling, occurrence, spatial-temporal distribution and fluxes. It is encouraged that future studies include both synthetic and none synthetic fibers.
\end{abstract}

Keywords: fibers, microplastics, Seine River, Paris agglomeration, plastics 


\section{Introduction}

La production de fibres mondiale a atteint 100 millions de tonnes en 2016 (1). Cette production comprend les fibres naturelles (coton), ainsi que les fibres chimiques, qu'elles soient artificielles (rayonne/viscose) ou synthétiques (polypropylène, polyamide, etc.). Les fibres synthétiques qui correspondent à des fibres de nature pétrochimiques représentent 65 millions de tonnes de cette production, tandis que les fibres artificielles, correspondant le plus souvent à de la cellulose régénérée, représentent 6,5 millions de tonnes. Quelle que soit leur nature, les filaments textiles produits à partir de fibres sont partie intégrante de notre quotidien et servent dans une multitude d'usage domestiques, en intérieur ou en extérieur : vêtements, literie, rideaux, tapisserie et tissus d'ameublement, mais aussi flexibles automobiles et matériaux de protection. Les filaments textiles sont aussi utilisés dans la pêche, en agriculture, en ingénierie civile (géotextiles) et plus généralement dans diverses industries (voitures, avions, etc.). L'omniprésence et l'usure des produits à base de fibres a pour conséquence un relargage considérable de microplastiques dans l'environnement.

La production de fibres augmente de $2 \%$ par année en moyenne et par voie de conséquence, elles sont de plus en plus présentes dans l'environnement. Le développement des recherches sur les microplastiques ces dernières années a permis d'attirer l'attention sur l'ubiquité des fibres dans les milieux aquatiques marins (2) et continentaux (3). Des études ont permis aussi de démontrer le nombre élevé de fibres introduites quotidiennement dans l'environnement à travers les rejets de stations d'épuration (4-6). Par ailleurs, des fibres ont aussi été retrouvées dans l'air intérieur de maisons et de bureaux, ainsi que dans l'air extérieur $(7,8)$.

Dû à leur faible rapport longueur/diamètre, les fibres sont facilement ingérables par les organismes (9-11). Ceci peut causer des dommages physiques le plus fréquemment liés à une perturbation du système digestif (blocage du tractus gastro-intestinal, fausses impressions de satiété, etc.) (12-16). La seconde catégorie de risques est liée au fait que les microplastiques soient porteurs d'un « cocktail de polluants » qu'ils peuvent transporter sur de longues distances et potentiellement relarguer au sein d'organismes les ayant ingérés. Ces polluants sont soit incorporés aux polymères durant la production (additifs) ou adsorbés sur leurs surfaces une fois dans l'environnement (17). Les fibres artificielles et naturelles sont aussi concernées. Notamment, des teintures utilisées en industrie textile sont cancérigènes (10). Ces fibres contiennent aussi fréquemment des retardateurs de flamme.

Malgré leur ubiquité et leur potentiel toxique, les études se concentrant uniquement sur les fibres sont très rares. Les travaux publiés sont le plus souvent orientés vers l'analyse de particules de forme irrégulières (fragments) ou sphériques. De plus, si les fibres sont inclues dans diverses études comme étant une souscatégories des microplastiques, les fibres naturelles et artificielles sont totalement négligées sauf dans de rares exceptions (9). Dans ce type d'étude, seules les fibres synthétiques sont considérées.

Dans ce contexte, il apparaissait nécessaire d'acquérir plus de données sur leur abondance dans l'environnement, et cette étude s'est donc exclusivement concentrées sur les fibres, sans distinction de leur composition. Les fibres minérales ont été exclues ainsi que les fibres naturelles non anthropogéniques, c.à.d. des fibres naturelles non revêtues d'additifs ou de colorants. Le premier objectif de cette étude était méthodologique et portait sur l'échantillonnage de fibres en rivière. En effet, les diverses études portant sur les microplastiques en milieu continental ont transposé les méthodes utilisées en milieu marin sans qu'aucune validation ne soit effectuée. Pour combler ce déficit, la variabilité spatio-temporelle ainsi que la distribution des fibres sur la colonne d'eau ont été étudiées. Le second visait avoir des estimations sur les concentrations en fibres et fournir des premières estimations de flux annuels. Un suivi sur la Seine a été effectué durant 19 mois sur 5 sites, de l'amont à l'aval de Paris. A l'aide de ces données, une première estimation des flux de fibres en Seine a été fournie.

\section{Matériels et méthodes}

\subsection{Site d'étude}

La Seine est un fleuve long de $777 \mathrm{~km}$ de sa source, dans le département de la Côte d'Or, jusqu'à son embouchure située au Havre. Son bassin représente une superficie de $78650 \mathrm{~km}^{2}$ dont le climat est considéré comme pluvial océanique. Dans sa partie amont, le bassin de la Seine intègre la région Île-de-France (12 000 
$\mathrm{km}^{2}$ soit environ $15 \%$ du bassin de la Seine). Au cours de sa traversée, le fleuve va recevoir les eaux usées traitées générées pas les activités urbaines $\left(27 \mathrm{~m}^{3} . \mathrm{s}^{-1}\right.$ rejetés par les STEP parisiennes), faiblement diluées par le débit du fleuve (débit moyen annuel à Paris-Austerlitz : $301 \mathrm{~m}^{3} \cdot \mathrm{s}^{-1}$; Banque hydro : http://www.hydro.eaufrance.fr).

\subsection{Dispositif d'échantillonnage}

Les prélèvements ont été effectués à l'aide d'un filet à plancton (maille $80 \mu \mathrm{m}$ - section $725 \mathrm{~cm}^{2}$ ) utilisé horizontalement, couplé à un courantomètre à hélice (OTT - C2'10.150' - mesure de vitesses de courant entre 0,025 et $5 \mathrm{~m} \cdot \mathrm{s}^{-1}$ ) (Figure 1). Le courant est mesuré en triplicats de manière simultanée dans le but d'évaluer le volume d'eau échantillonné.

Afin d'éviter le risque de colmatage du filet, lié à la présence de MES en Seine des tests préliminaires ont été effectués, et lors des campagnes, les volumes prélevés sont restés systématiquement en deçà du seuil de colmatage estimé à $8 \mathrm{~m}^{3} / \mathrm{s}$ pour des concentrations en MES usuelles inférieures à $40 \mathrm{mg} / \mathrm{L}$ Seule la couche superficielle de la colonne d'eau a été considérée $(0,1-0,35 \mathrm{~m})$.

Après chaque prélèvement, le filet est rincé trois fois par l'extérieur avec de l'eau de rivière. L'efficacité du rinçage a été testée lors d'un prélèvement avant le début du suivi. Le quatrième rinçage n'augmente plus la quantité de fibres collectée.

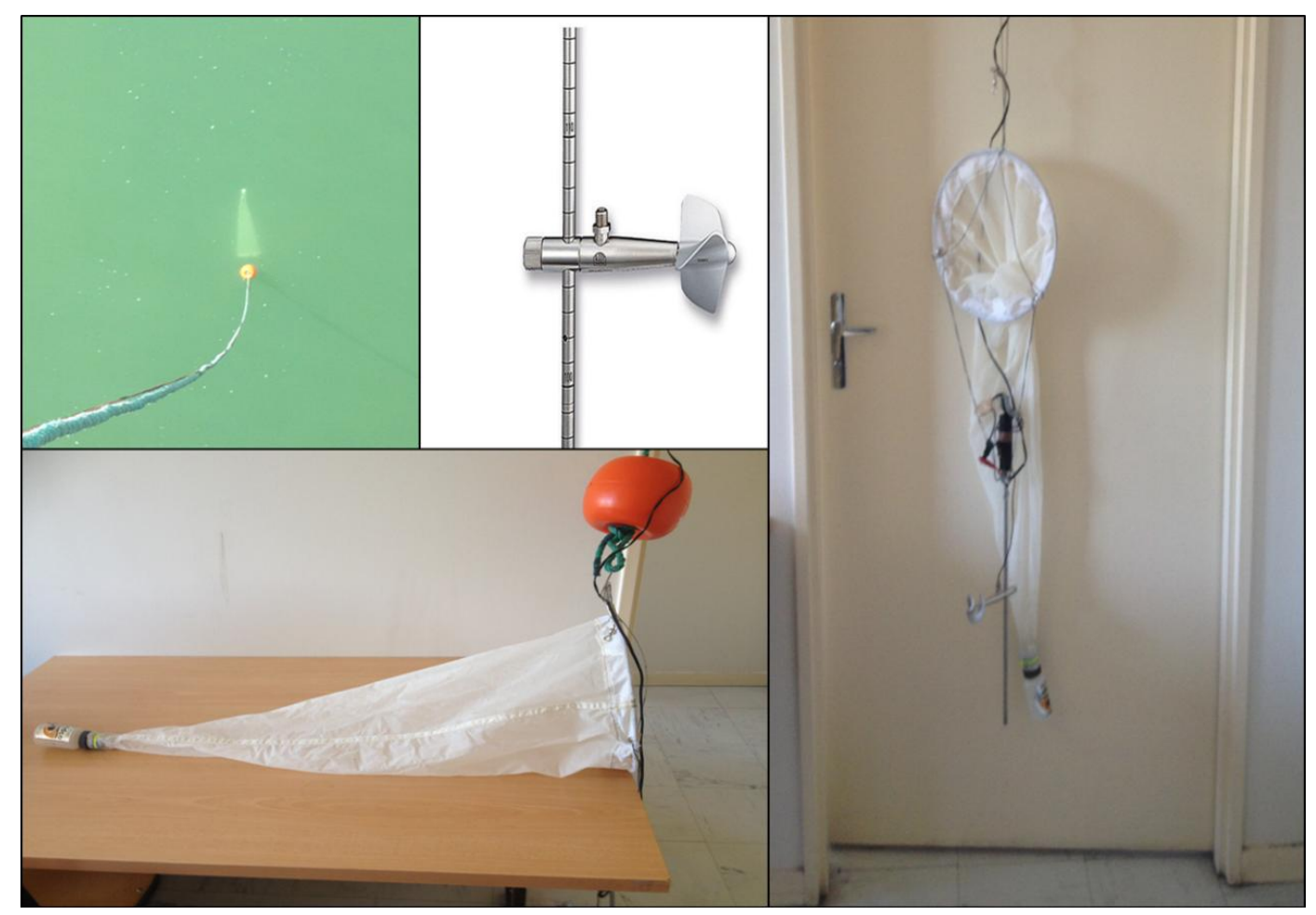

Figure 1 : Filet à plancton de maille 80 um couplé à un courantomètre à hélice

\subsection{Variabilité temporelle}

Deux campagnes ont été effectuées dans le but de déterminer la variabilité temporelle des concentrations sur 2 heures des concentrations en microplastiques. Lors de la première campagne le 12 mars 2015 sur le site P1, 6 prélèvements ont été réalisés dans une période de $2 \mathrm{~h}(10 \mathrm{~h} 52$ à $12 \mathrm{~h} 48)$. Chaque prélèvement a duré 1 minute et le volume échantillonné était en moyenne de $2,21 \mathrm{~m}^{3}$ avec un débit mesuré de $144 \mathrm{~m}^{3} \cdot \mathrm{s}^{-1}$.

La deuxième campagne a été réalisée sur le même site le 6 juillet 2015. Les 6 prélèvements ont cette fois duré 3 minutes du fait du débit plus faible de la rivière $\left(24 \mathrm{~m}^{3} \cdot \mathrm{s}^{-1}\right)$ qui a permis d'augmenter la durée de prélèvement sans colmatage. Le volume moyen d'échantillonnage était de $2,72 \mathrm{~m}^{3}$. Le premier prélèvement a été effectué à $12 \mathrm{~h} 03$ et le dernier à $15 \mathrm{~h} 00$. 


\subsection{Variabilité spatiale à travers la section}

À la station P1, des prélèvements ont été effectués en triplicats en cinq points de la section de la rivière le 23 avril 2015 (débit de $69 \mathrm{~m}^{3} \cdot \mathrm{s}^{-1}$, exposition de 3 minutes). Les échantillons ont été prélevés juste sous la surface (rive droite, milieu, rive gauche) et à différentes profondeurs au milieu de la rivière (Figure 2). La profondeur maximale a été évaluée à $2,6 \mathrm{~m}$ à l'aide d'un échosondeur. Tous les prélèvements ont été effectués sur une durée totale de 3 heures.

$67 \mathrm{~m}$

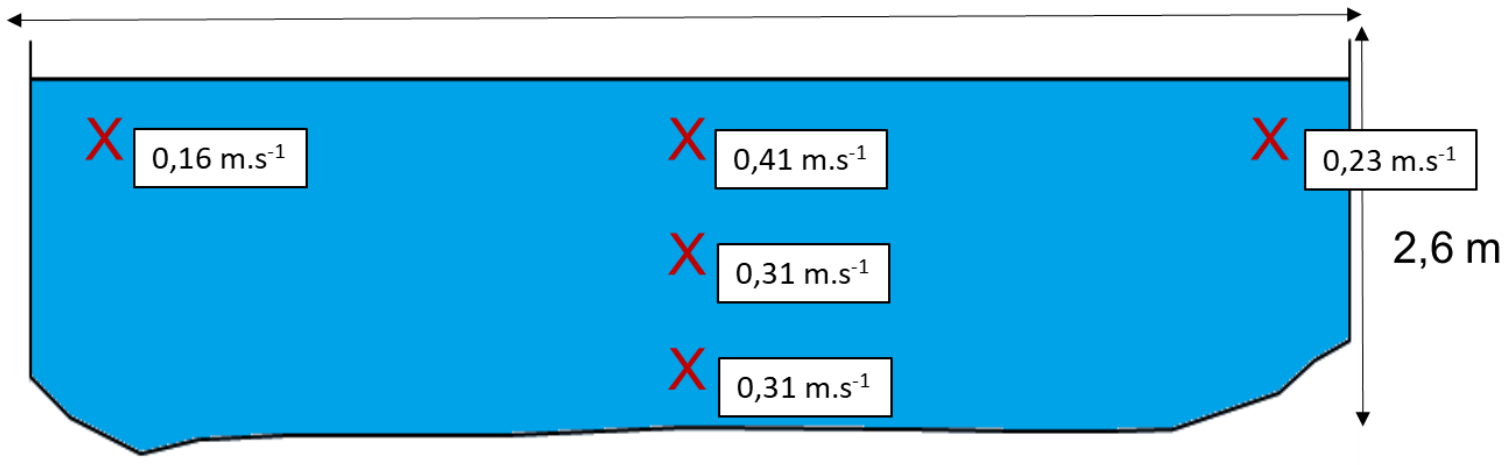

Figure 2 : Points de prélèvement sur la section de la rivière avec les courants mesurés correspondants.

\subsection{Suivi dans la Seine et la Marne}

Une fois par mois, d'avril 2014 à décembre 2015 et en collaboration avec la Direction de la Prospective et du Développement du SIAAP, des prélèvements ont été effectués sur quatre stations sur la Seine en amont et en aval de Paris (P2-P5) ainsi que sur une station en Marne (P1) (Figure 3). Deux campagnes de prélèvement n'ont pas pu avoir lieu, en janvier et août 2015, ce qui a donné un total de 19 campagnes. Les échantillonnages ont toujours été réalisés sur une même journée pour les cinq stations, en milieu de section, à partir de ponts. Les volumes échantillonnés variaient de 0,2 à $4,0 \mathrm{~m}^{3}$ et le débit a varié de 21 à $856 \mathrm{~m}^{3} \cdot \mathrm{s}^{-1}$.

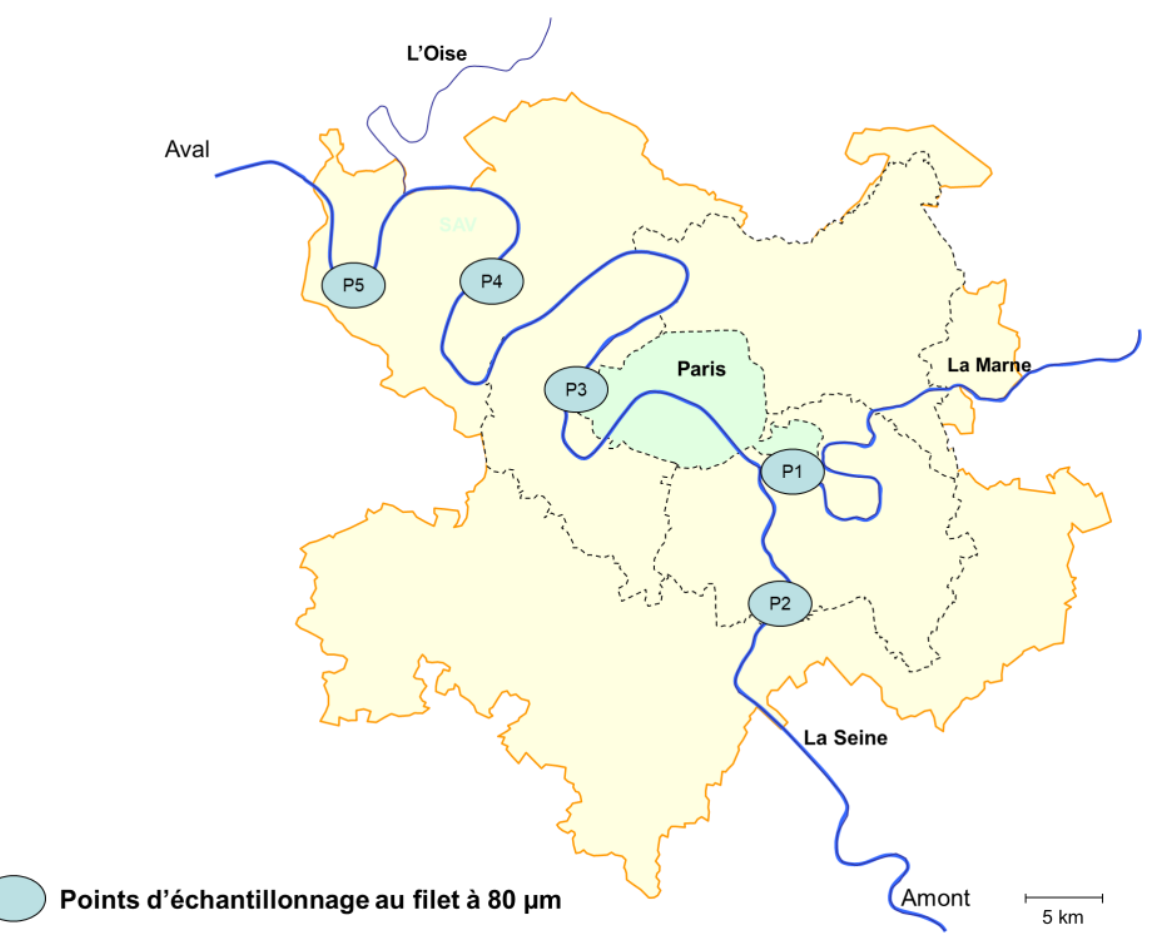

Figure 3 : Emplacement des sites d'échantillonnage durant le suivi annuel. 


\subsection{Traitement des échantillons}

Lors de la manipulation des échantillons, uniquement de la verrerie en verre préalablement grillée et de l'acier inoxydable ont été utilisés, les échantillons étaient couverts d'aluminium et une blouse en coton était portée pour limiter les risques de contamination.

Afin de purifier les échantillons avant l'observation, $1 \mathrm{~g}$ de Sodium Dodecyl Sulfate (SDS) a été ajouté à chaque échantillon, placé à l'étuve à $70^{\circ} \mathrm{C}$ pendant 24 heures. Ensuite, $1 \mathrm{~mL}$ de bioenzymes $\mathrm{SE}$ et $\mathrm{F}$ (lipases, amylases, protéases) a été introduit dans les échantillons replacés à l'étuve à $40^{\circ} \mathrm{C}$ pour $48 \mathrm{~h}$. Après ajout de $15 \mathrm{~mL}$ de peroxyde d'hydrogène $\left(\mathrm{H}_{2} \mathrm{O}_{2}\right)$, les échantillons sont remis à l'étuve à $40^{\circ} \mathrm{C}$ pendant $48 \mathrm{~h}$. Certains échantillons étant très chargés en matières en suspension, une séparation densimétrique a été réalisée à l'aide d'une solution saturée de chlorure de zinc $\left(\mathrm{ZnCl}_{2}\right.$, densité $\left.=1,6426 \pm 0,0016 \mathrm{~g} . \mathrm{cm}^{-3}\right)$. Finalement, les échantillons ont été filtrés sur des filtres en fibre de verre GF/D Whatman d'un diamètre de $47 \mathrm{~mm}$ et d'une porosité de $2,7 \mu \mathrm{m}$.

\subsection{Quantification et caractérisation}

Les filtres sont observés à un grossissement x 16 avec un stéréo-microscope Leica MZ12 couplé à un logiciel d'analyse d'image. Les fibres peuvent ainsi être repérées et comptabilisées. Puisque certaines fibres peuvent être aussi d'origine naturelle, la reconnaissance de fibres anthropogéniques est établie grâce à des critères établis dans la littérature $(18,19)$. Le logiciel Histolab, utilisé en couplage avec la loupe, permet aussi de mesurer la longueur des fibres. Ces dernières ont ainsi été classées par des intervalles de tailles de $200 \mu \mathrm{m}$ entre 50 et $5000 \mu \mathrm{m}$.

Afin d'identifier la nature chimique des fibres observées et avoir une estimation de la proportion des fibres synthétiques - c.à.d. celles considérées comme des microplastiques - la caractérisation d'un sous-échantillon de 25 fibres a été effectuée à l'aide d'un micro-spectroscope IRTF-RTA. Les spectres obtenus pour chaque fibre sont comparés à une base de données permettant de terminer le polymère constituant la fibre.

\section{Résultats}

\subsection{Description morphologique et nature des fibres}

Des fibres de différentes couleurs ont été observées avec une prédominance des fibres bleues. Le diamètre moyen des fibres est de $25 \mu \mathrm{m}$ mais peut varier de 5 à $100 \mu \mathrm{m}$. La distribution en longueur des fibres suit une tendance décroissante. La classe de taille [250-450 $\mu \mathrm{m}$ ] est la plus abondante (Figure 4), alors que l'importance des classes de taille supérieure décroît. La plus petite classe de taille [50-250 $\mu \mathrm{m}]$ contient moins de fibres, ce qui pourrait être dû au fait que certaines fibres passent facilement à travers les mailles du filet $80 \mu \mathrm{m}$. 


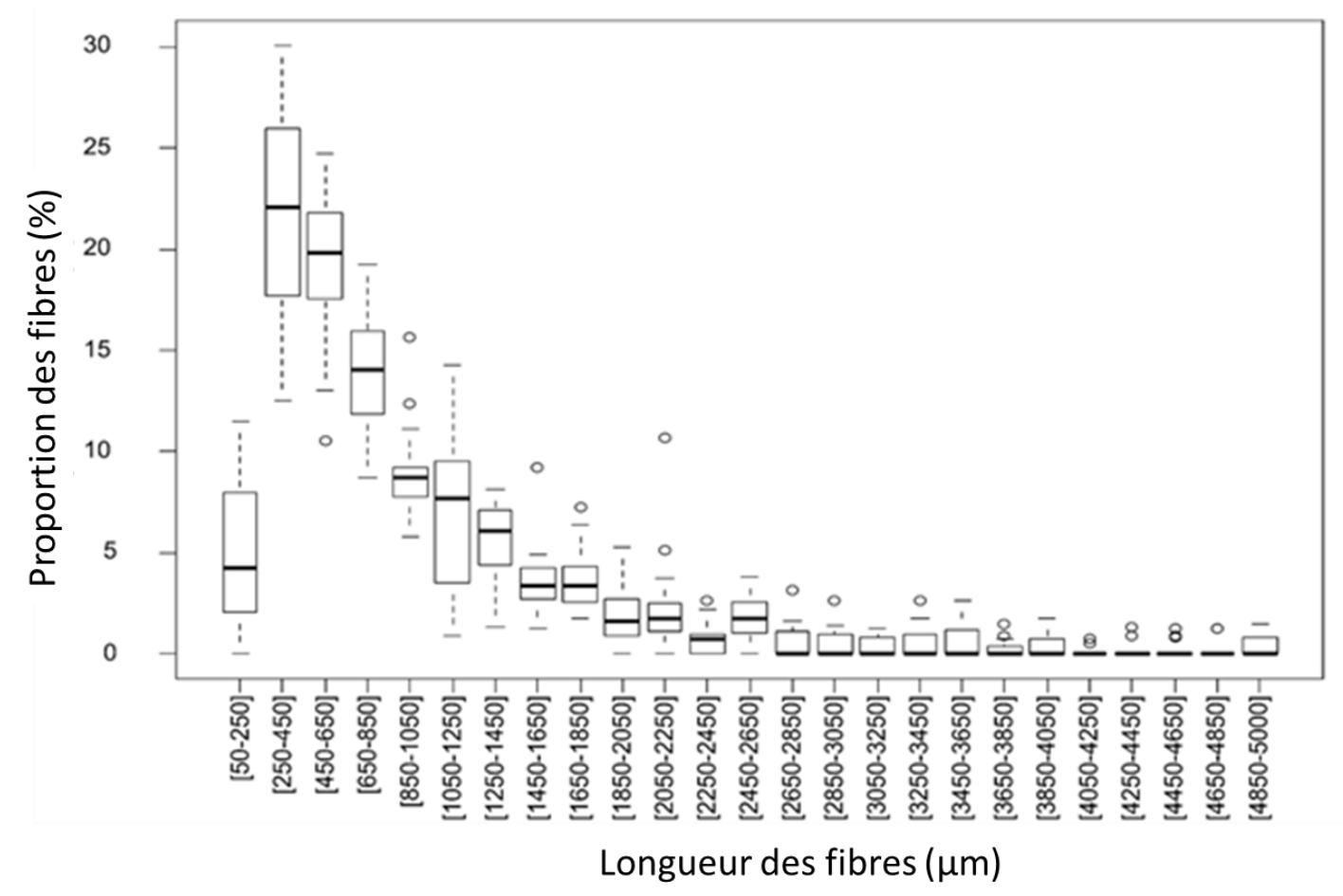

Figure 4 : Distribution en longueur des fibres dans les échantillons de rivière (avec $n=15$ échantillons et 1664 fibres mesurées). Les boîtes à moustache sont tracées, de bas en haut, de la façon suivante : [ Quartile inférieur - 1,5*Ecart interquartile ], [ Quartile inférieur ], [ Médiane ], [ Quartile supérieur ], [ Quartile supérieur $+1,5^{*}$ Ecart interquartile ]. Les valeurs extrêmes sont représentées par des points isolés.

D'après les spectres, 15 fibres correspondent à de la rayonne et une fibre à du coton. Cette dernière correspondance pourrait être remise en question car les spectres de la rayonne et du coton sont très similaires. Les fibres restantes ( 9 fibres soit $65 \%$ des fibres analysées) sont de nature synthétique. Cinq de ces fibres ont été caractérisées comme du polyéthylène téréphtalate, deux comme du polypropylène et une comme du polyamide. La dernière fibre est un mélange de polyéthylène téréphtalate et polyuréthane. Le polyéthylène téréphtalate est le polymère le plus utilisé pour les fibres synthétiques. Ces résultats illustrent le fait qu'on retrouve un mélange de fibres dans les eaux de surface (Figure 5).
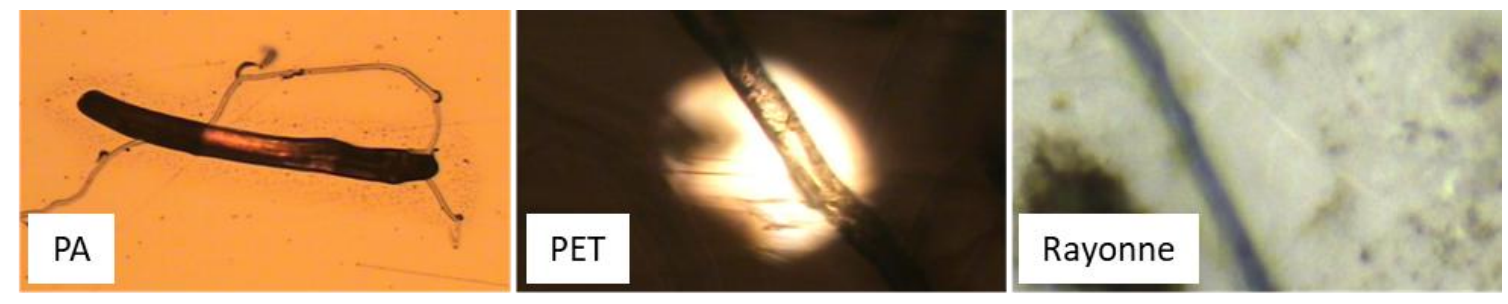

Figure 5 : Exemples de fibres identifiées à l'aide de la micro-spectroscopie IRTF.

\subsection{Variabilité temporelle à court terme}

Lors de la première campagne de prélèvement, les concentrations variaient de 38,2 à 101,6 fibres.m ${ }^{-3}$ (Figure 6) avec un coefficient de variation de $45 \%$ (avec $n=6$ ). Cette variabilité semble particulièrement élevée et pourrait être attribuée à la courte période d'exposition du filet $(1 \mathrm{~min})$ ou à des variations de concentrations très importantes. Pour tester ces hypothèses, le temps d'exposition du filet a été triplé pour la deuxième campagne ( 3 minutes au lieu d'1 minute). Les concentrations étaient alors entre 18,7 et 38,6 fibres. $\mathrm{m}^{-3}$ avec une concentration moyenne de 30,4 fibres. $\mathrm{m}^{-3}$ et un coefficient de variation de seulement $26 \%(n=6)$. Un débit bien plus faible à la seconde campagne peut aussi expliquer cette différence. 


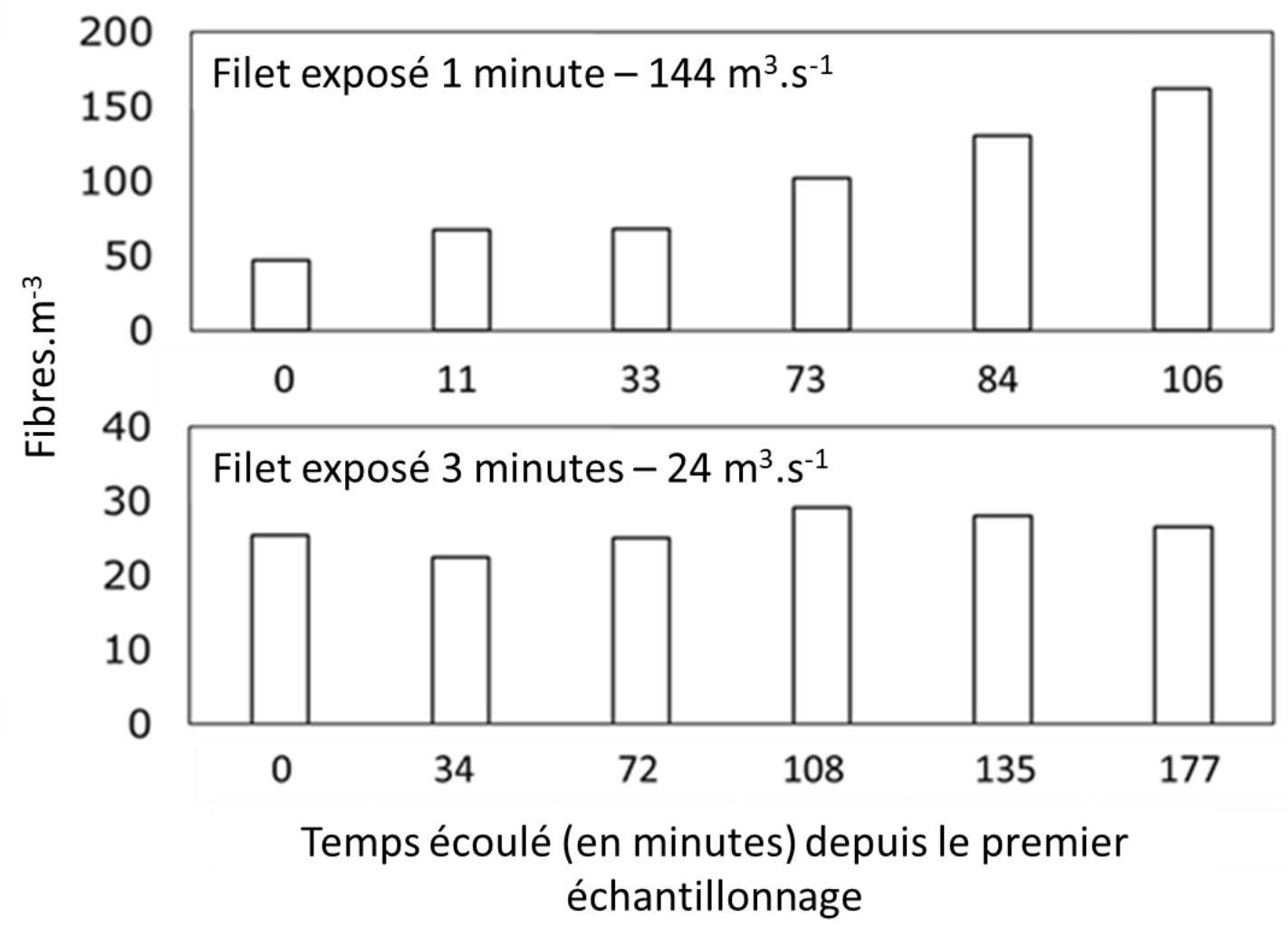

Figure 6 : Variabilité à court terme des concentrations en fibres au cours des deux campagnes effectuées
respectivement en deux et trois heures.

\subsection{Variabilité spatiale à travers la section}

Des triplicats d'échantillons de surface de microplastiques au milieu de la rivière ont montré des concentrations de 16,8 - 21,3 - 24,7 fibres.m ${ }^{-3}$ (Figure 7). Des concentrations plus importantes ont été observées proches des berges avec 52,4 - 61,0 - 87,7 fibres. $\mathrm{m}^{-3}$ pour la rive gauche et $32,1-34,8-46,4$ fibres. $\mathrm{m}^{-3}$ pour la rive droite. Le coefficient de variation pour les 9 échantillons de surface après une exposition de trois minutes est de 53\%, ce qui est deux fois plus élevé que le coefficient de variation retrouvé pour la variabilité temporelle sur 2 heures. Cela montre une différence de concentrations potentielle entre le centre de la rivière et les rives.

Les concentrations plus élevées observées à proximité des rives pourraient être liées à l'intense trafic fluvial de l'agglomération parisienne. En effet, on observe aisément que les macroplastiques et autres débris flottants sont repoussés vers les rives par les vagues formées lors du passage de nombreux bateaux. Les microplastiques pourraient subir ce même phénomène. La concentration de microplastiques à proximité des berges est sans douté renforcée par les plus faibles courants observés comparativement au centre de la rivière.

Des concentrations de 19,0 - 20,2 - 28,2 fibres. $\mathrm{m}^{-3}$ ont été retrouvées à $1 \mathrm{~m}$ de profondeur et 13,7 - 16,9 19,1 fibres. $\mathrm{m}^{-3}$ à $2 \mathrm{~m}$ de profondeur. En prenant en compte les neuf échantillons collectés au milieu de la section à trois profondeurs différentes, le coefficient de variation calculé pour la variabilité verticale (21\%) est plus de deux fois inférieur à celui estimé pour la variabilité horizontale. Dans les études en environnement marin, les filets flottants sont très utilisés. S'il peut être facilement concevable que les fibres et les microplastiques flottent en milieu marin, les conditions hydrodynamiques en rivière pourraient induire une répartition différente à travers la section. L'absence d'hétérogénéité verticale dans la rivière est probablement due à la turbulence ainsi qu'au fait que la densité de l'eau douce est plus faible que celle du milieu marin. La navigation contribue également à la turbulence. 


\section{$67 \mathrm{~m}$}

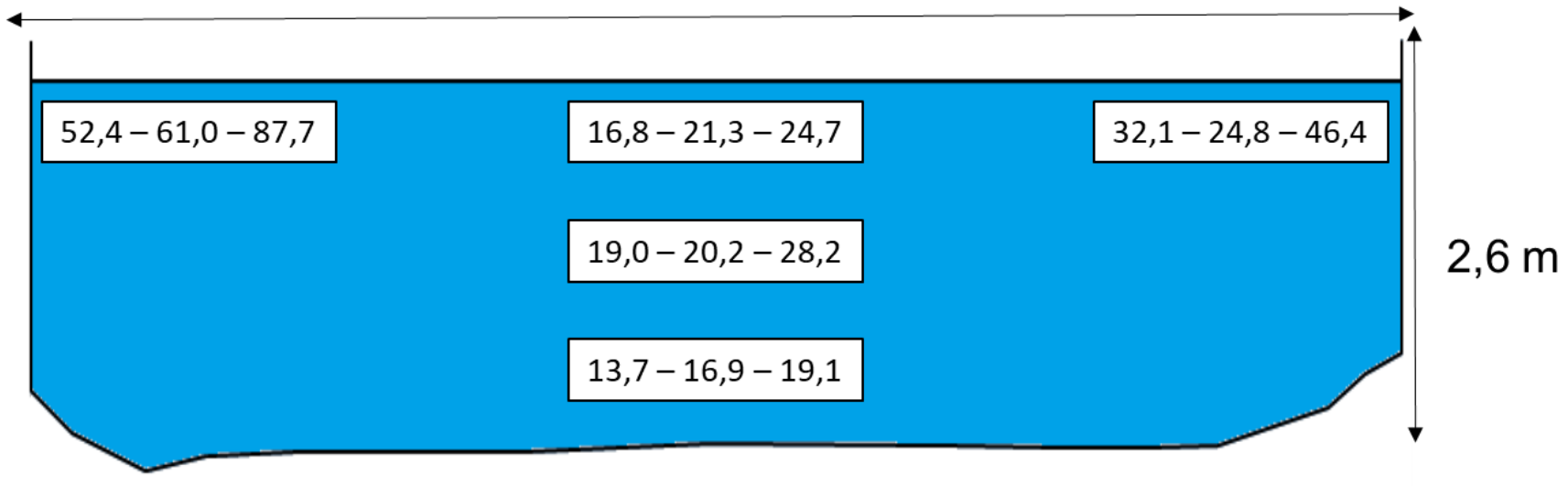

Figure 7 : Concentrations en fibres, exprimées en fibres.m ${ }^{-3}$, pour les 5 points échantillonnés.

\subsection{Suivi dans la Seine et la Marne}

Les concentrations durant le suivi dans la Marne (P1) se situent entre 5,7 et 398,0 fibres. $\mathrm{m}^{-3}$ avec une concentration moyenne de 100,6 \pm 99,9 fibres. $\mathrm{m}^{-3}$ (moyenne \pm écart type). Pour la Seine, les concentrations observées de l'amont à l'aval sont de 48,5 \pm 98,5 fibres. $\mathrm{m}^{-3}$ (P2), 27,9 \pm 26,3 fibres. $\mathrm{m}^{-3}$ (P3), 27,9 \pm 40,3 fibres. $\mathrm{m}^{-3}(\mathrm{P} 4)$ et $22,1 \pm 25,3$ fibres. $\mathrm{m}^{-3}$ (P5). Les résultats sont présentés en détails Figure 8.

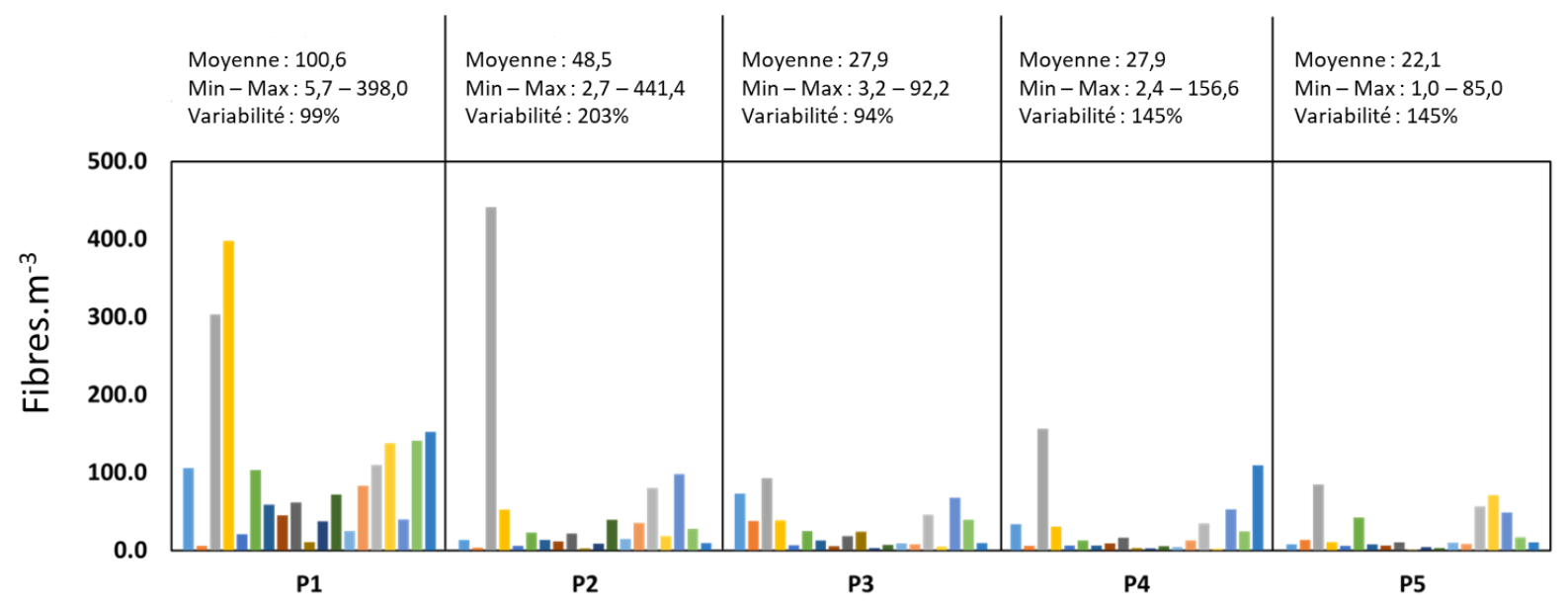

Figure 8: Concentrations observées pour les 5 sites pendant le suivi.

Les concentrations varient fortement comme en témoigne le coefficient de variation qui varie entre $94 \%$ et $203 \%$. Cette variabilité est bien supérieure à la variabilité temporelle à court terme. Les variations semblent survenir aux mêmes dates sur les différents sites. En testant la corrélation entre les sites, il apparait que les différents sites présentent des niveaux de concentration corrélés (sauf entre P1 et P3 - corrélation de spearman). Cela pourrait montrer que des facteurs globaux variant de la même façon sur tous les sites peuvent davantage affecter les concentrations que des facteurs locaux et ponctuels. Des changements d'apports diffus ou des variations saisonnières pourraient en être la cause, mais il pourrait également y avoir un lien avec les variations de débit.

Une analyse statistique montre qu'il n'y a pas de corrélation significative entre le débit de la rivière et les concentrations aux différents sites $\left(0,09<\mathrm{R}^{2}<0,27\right)$. On observe cependant une certaine tendance pour les périodes de forts débits pendant lesquelles les niveaux de fibres semblent plutôt faibles. Lors des périodes de bas débits, les niveaux de concentration sont plus variables et pourraient être influencés par différents paramètres tels que l'apport de fibres par des sources ponctuelles (STEP, DO), des sources diffuses (retombées atmosphériques) ou une remise en suspension possible de fibres à partir des sédiments. 
Une comparaison des sites (test de Mann-Whitney) montre que les concentrations ne sont pas significativement différentes à l'exception du site $\mathrm{P} 1$. Ce point présentant des concentrations significativement plus élevées est le seul situé sur la Marne, ce qui est cohérent avec la tendance observée montrant des concentrations plus élevées lors de bas débits. Les connaissances actuelles ne permettent pas d'expliquer une telle différence.

Au vu des différents apports de fibres potentiels localisés entre les sites de prélèvement, le fait que les niveaux de concentration ne soient pas significativement différents entre l'amont et l'aval semble contre intuitif. En effet entre P2 et P5, la Seine croise deux affluents (la Marne et l'Oise), trois stations d'épuration dont la plus importante de France (Seine Amont, Seine Centre et Seine Aval), des déversoirs d'orage et la ville de Paris avec sa population très dense. L'absence de différence entre l'amont et l'aval de Paris pourrait être expliquée par la compensation de ces apports par différents puits tels que la sédimentation et/ou le dépôt de fibres sur les berges. La dégradation n'a pas été envisagée comme une explication probable étant donné que le temps de transit des particules entre ces points de prélèvement d'environ 72 heures est bien plus faible que le temps nécessaire à leur dégradation.

\subsection{Flux annuels en Seine}

Il est possible d'estimer les flux annuels de fibres dans la Seine d'après les 19 flux ponctuels calculés pour chaque site. Les flux moyens du point P1 au point P5 sont de $1.4 \times 10^{11}, 1.7 \times 10^{11}, 1.6 \times 10^{11}, 1.3 \times 10^{11}$ et $1.8 \times 10^{11}$ fibres par an respectivement. Le point amont $\mathrm{P} 2$ et le point aval $\mathrm{P} 5$ révèlent des flux très proches. L'augmentation entre les deux stations est de seulement $6 \%$, ce qui est bien plus faible que l'incertitude induite par la variabilité à court terme présentée précédemment. Par conséquent, l'impact de l'agglomération parisienne ne peut pas être mis en évidence pour ce qui est des fibres et les connaissances actuelles ne permettent pas de comprendre et d'expliquer cette absence de tendance. On pourrait supposer que dans le cas de l'agglomération parisienne les puits et les sources présentent des niveaux similaires. Les flux maximum et minimum estimés pour le site P5 sont de $2,8 \times 10^{10}$ et $6,1 \times 10^{11}$ fibres par an avec une moyenne de $1,8 \times 10^{11}$.

\section{Conclusions}

Cette étude montre les fibres, qu'elles soient synthétiques ou non, présentent des concentrations élevées en rivière. Comme pour les fibres plastiques, les fibres non synthétiques contiennent divers colorants et additifs, dont certains ont pour but de ralentir leur dégradation. L'impact exact de ces fibres doit encore être identifié. En considérant leur possible impact environnemental ainsi que le fait que leur sources et dynamiques en rivières sont similaires à ceux des fibres synthétiques, il serait pertinent de les inclure systématiquement dans les études futures.

Cet article fournit des nouvelles informations sur les variabilités spatiales et temporelles ainsi que des données les concentrations et flux de fibres à l'échelle de l'agglomération parisienne. Nous conseillons pour les futures études souhaitant analyser les fibres (couplées à d'autres types de microplastiques ou séparément) d'utiliser un filet avec un maillage à $80 \mu \mathrm{m}$ par opposition au maillage classiquement utilisé de $300 \mu \mathrm{m}$. Ceci permet de réduire considérablement la proportion de fibres passant à travers le filet donnant ainsi un échantillonnage plus représentatif. L'utilisation d'un maillage faible pose un problème de colmatage du filet obligeant à échantillonner des volumes plus faibles. Même si cette étude a démontré qu'un échantillonnage plus long (3 minutes vs. 1 minute) permet de réduire la variabilité, le colmatage empêche d'augmenter les temps d'échantillonnage, surtout en période de crue. Plus de connaissances sont nécessaires afin de déterminer l'équilibre parfait entre le temps d'échantillonnage et la représentativité des échantillons.

La variabilité des fibres à travers la section a aussi été déterminée, montrant une homogénéité verticale tandis que les concentrations augmentent près des berges. Compte tenu du temps nécessaire à l'analyse des microplastiques, il est compliqué de multiplier le nombre d'échantillons lors d'un suivi environnemental. Le choix qui a été effectué dans ce premier travail a été donc d'échantillonner systémiquement en surface et au centre de la section. Ainsi, il doit être noté que les résultats présentés au niveau du suivi ne tiennent pas compte de la variabilité horizontale, affectant notamment les estimations de flux.

Lors du suivi mensuel sur 5 sites, les concentrations ont évolué de manière similaire sur tous les sites. Ceci- 
dit, aucune corrélation directe entre le débit et les concentrations n'a pu être mise en évidence. Il apparait ainsi que même si les concentrations sont partiellement affectées par les débits, d'autres facteurs encore non déterminés entrent en jeu. Ces derniers sont potentiellement liés aux sources mais aussi aux puits. En effet, les concentrations à l'amont et à l'aval de Paris sont similaires suggérant une présence de puits important pouvant contrebalancer l'apport de fibres. La sédimentation est une des pistes majeures qui doivent être explorées dans les études futures.

Cette étude apporte des données inédites sur les fibres dans un milieu fluvial, leur abondance, leur variabilité, leur distribution, et leurs flux. Ceci-dit, le manque actuel de compréhension sur la dynamique des fibres dans l'environnement ainsi que la faible quantité de données présentes dans la littérature limitent les possibilités d'interprétation des résultats. Il est nécessaire dans le futur d'identifier le rôle de différents mécanismes tel que la sédimentation, la re-supension, la fragmentation et le dépôt sur les berges. De plus, ces différents mécanismes vont affecter de manières différentes les microplastiques, dépendant de leur taille, forme (fibres vs. fragments), composition en polymères, densité, présence et quantité de divers additifs etc. rendant ainsi la compréhension plus complexe. Une approche de modélisation pourrait aider à surmonter ces obstacles.

Néanmoins, ce travail a permis d'acquérir des connaissances précieuses. Il a permis de démontrer l'abondance des fibres dans la Seine et la Marne, qu'elles soient de nature végétale (coton, laine, etc.), artificielle (rayonne) ou synthétiques (polyamide, polypropylène etc.). Ainsi, en tenant compte du fait que cet ensemble de fibres est présent en association avec d'autres polluants chimiques, il est conseillé pour les études environnementales futures de considérer tous les différents types de fibres, et non pas seulement celles labélisées comme étant des microplastiques, au vu de leur nature pétrochimique. Ceci est particulièrement vrai pour les expérimentations en écotoxicologie. En effet l'étendu du potentiel impact de ces fibres est encore très mal connu et il est urgent de mieux le cerner. 


\section{Bibliographie :}

1. International Cotton Advisory Committee. World Textile Demand Report: world consumption of major textile fibers. 2017 avr.

2. Lusher AL, Tirelli V, O'Connor I, Officer R. Microplastics in Arctic polar waters: the first reported values of particles in surface and sub-surface samples. Sci Rep. 8 oct 2015;5:14947.

3. Mani T, Hauk A, Walter U, Burkhardt-Holm P. Microplastics profile along the Rhine River. Sci Rep. 8 déc 2015;5:17988.

4. Murphy F, Ewins C, Carbonnier F, Quinn B. Wastewater Treatment Works (WwTW) as a Source of Microplastics in the Aquatic Environment. Environ Sci Technol. 7 juin 2016;50(11):5800- 8.

5. Talvitie J, Mikola A, Setälä O, Heinonen M, Koistinen A. How well is microlitter purified from wastewater? - A detailed study on the stepwise removal of microlitter in a tertiary level wastewater treatment plant. Water Res. 1 févr 2017;109:164- 72.

6. Browne MA, Crump P, Niven SJ, Teuten E, Tonkin A, Galloway T, et al. Accumulation of Microplastic on Shorelines Woldwide: Sources and Sinks. Environ Sci Technol. nov 2011;45(21):9175- 9.

7. Dris R, Gasperi J, Mirande C, Mandin C, Guerrouache M, Langlois V, et al. A first overview of textile fibers, including microplastics, in indoor and outdoor environments. Environ Pollut. févr 2017;221:453- 8 .

8. Dris R, Gasperi J, Saad M, Mirande C, Tassin B. Synthetic fibers in atmospheric fallout: A source of microplastics in the environment? Mar Pollut Bull [Internet]. 2016;(Query date: 2017-02-02). Disponible sur: http://www.sciencedirect.com/science/article/pii/S0025326X16300066

9. Lusher AL, McHugh M, Thompson RC. Occurrence of microplastics in the gastrointestinal tract of pelagic and demersal fish from the English Channel. Mar Pollut Bull. févr 2013;67(1- 2):94- 9.

10. Remy F, Collard F, Gilbert B, Compère P, Eppe G, Lepoint G. When Microplastic Is Not Plastic: The Ingestion of Artificial Cellulose Fibers by Macrofauna Living in Seagrass Macrophytodetritus. Environ Sci Technol. 15 sept 2015;49(18):11158- 66.

11. Sanchez W, Bender C, Porcher J-M. Wild gudgeons (Gobio gobio) from French rivers are contaminated by microplastics: Preliminary study and first evidence. Environ Res. janv 2014;128:98- 100 .

12. Farrell P, Nelson K. Trophic level transfer of microplastic: Mytilus edulis (L.) to Carcinus maenas (L.). Environ Pollut. juin 2013;177:1- 3.

13. Derraik JG. The pollution of the marine environment by plastic debris: a review. Mar Pollut Bull. 2002;44(9):842-852.

14. Carr KE, Smyth SH, McCullough MT, Morris JF, Moyes SM. Morphological aspects of interactions between microparticles and mammalian cells: intestinal uptake and onward movement. Prog Histochem Cytochem. 2012;46(4):185- 252.

15. Cole M, Lindeque P, Fileman E, Halsband C, Goodhead R, Moger J, et al. Microplastic Ingestion by Zooplankton. Environ Sci Technol. 6 juin 2013;130606145528005.

16. Tourinho PS, Ivar do Sul JA, Fillmann G. Is marine debris ingestion still a problem for the coastal marine biota of southern Brazil? Mar Pollut Bull. mars 2010;60(3):396- 401. 
17. Rochman CM, Browne MA. Classify plastic waste as hazardous. Nature. 14 févr 2013;494(7436):169- 71.

18. Hidalgo-Ruz V, Gutow L, Thompson RC, Thiel M. Microplastics in the Marine Environment: A Review of the Methods Used for Identification and Quantification. Environ Sci Technol. 20 mars 2012;46(6):3060- 75 .

19. Norén F. Small plastic particles in Coastal Swedish waters - Kimo reports. http://www.kimointernational.org/WebData/Files/Small\%20plastic\%20particles\%20in\%20Swedish\%2 0West $\% 20$ Coast $\% 20$ Waters.pdf.; 2007. 11 p. 